\title{
Modeling extreme wave heights from laboratory experiments with the nonlinear Schrödinger equation
}

\author{
H. D. Zhang ${ }^{1}$, C. Guedes Soares ${ }^{1}$, Z. Cherneva ${ }^{1}$, and M. Onorato ${ }^{2}$ \\ ${ }^{1}$ Centre for Marine Technology and Engineering (CENTEC), Instituto Superior Técnico, Technical University of Lisbon, \\ Lisbon, Portugal \\ ${ }^{2}$ Dipartimento di Fisica, Universitá di Torino, Via P. Giuria 1, 10125 Torino, Italy
}

Correspondence to: C. Guedes Soares (guedess@mar.ist.utl.pt)

Received: 23 June 2013 - Published in Nat. Hazards Earth Syst. Sci. Discuss.: 8 October 2013

Revised: 23 August 2013 - Accepted: 4 March 2014 - Published: 24 April 2014

\begin{abstract}
Spatial variation of nonlinear wave groups with different initial envelope shapes is theoretically studied first, confirming that the simplest nonlinear theoretical model is capable of describing the evolution of propagating wave packets in deep water. Moreover, three groups of laboratory experiments run in the wave basin of CEHIPAR (Canal de Experiencias Hidrodinámicas de El Pardo, known also as El Pardo Model Basin) was founded in 1928 by the Spanish Navy. are systematically compared with the numerical simulations of the nonlinear Schrödinger equation. Although a little overestimation is detected, especially in the set of experiments characterized by higher initial wave steepness, the numerical simulation still displays a high degree of agreement with the laboratory experiments. Therefore, the nonlinear Schrödinger equation catches the essential characteristics of the extreme waves and provides an important physical insight into their generation. The modulation instability, resulting from the quasi-resonant four-wave interaction in a unidirectional sea state, can be indicated by the coefficient of kurtosis, which shows an appreciable correlation with the extreme wave height and hence is used in the modified Edgeworth-Rayleigh distribution. Finally, some statistical properties on the maximum wave heights in different sea states have been related with the initial Benjamin-Feir index.
\end{abstract}

\section{Introduction}

In the past, the free surface elevation in deep water is assumed to follow a Gaussian structure and is modeled as the linear superposition of a large number of elementary wavelets with Rayleigh distributed amplitudes and random phases (Longuet-Higgins, 1952). At this linear level of approximation, surface displacements are symmetric with respect to the mean water level and completely described by the autocorrelation function or power spectrum.

However, surface waves are nonlinear in nature and bound waves need to be taken into account (Hasselmann, 1962; Longuet-Higgins, 1963). As reviewed by Guedes Soares (2003), numerous theoretical and empirical models of wave heights have been suggested after the proposal of the linear Rayleigh model. The non-Gaussian and phase-coupled bound modes have no significant effect on the crest-to-trough wave heights although they make wave crests shaper and narrower, and troughs shallower and more rounded (Tayfun and Fedele, 2007; Petrova et al., 2008).

In a recent study, third-order nonlinearity represented by the fourth-order cumulants has been successfully applied in the statistics of the so-called abnormal, freak or rogue waves, of which the probability is described reasonably well by the theoretical approximation based on Gram-Charlier (GC) expansions (Tayfun and Fedele, 2007). The deviation from Gaussian structure is mainly attributed to modulation instability in the wave train, which can be considered as a quasiresonant four-wave interaction in unidirectional narrowband waves. The third-order nonlinear interactions between free wave modes, described quantitatively by means of the coefficient of kurtosis $\lambda_{40}$, are responsible for the large amplitude events and the increased probability of occurrence of abnormal waves, as shown in a series of studies (e.g., Hagen, 2002; Guedes Soares et al., 2003, 2004a, b; Petrova et al., 2007; 
Onorato et al., 2006; Mori et al., 2007; Cherneva et al., 2009, 2011; Shemer et al., 2009, 2010).

Due to the potentially severe damage from extreme waves to the vessels and offshore structures at sea (Guedes Soares et al., 2008; Fonseca et al., 2010), a lot of efforts have been devoted to the topic of the mechanisms of their generation. A number of reasons why freak wave phenomena may occur have been explained. Besides the linear superposition of Fourier components with coherent phases and strong wavecurrent interaction or wave diffraction, recently it has been strongly argued that nonlinear self-modulation of a slowly varying wave train can produce abnormal waves. A simple example is the case of a uniform narrowband wave train to sideband perturbations where nonlinear interaction known as the Benjamin-Feir instability will result in focusing of wave energy in space and/or time as illustrated in laboratory experiments (Lake et al., 1977).

To a first approximation, the evolution of the envelope of a narrowband wave train is described by the nonlinear Schrödinger equation, which was first derived by Zakharov (1968) using a spectral method and by Hasimoto and Ono (1972) and Davey (1972) using multiple-scale methods. The nonlinear Schrödinger equation in one-space dimension may be solved by means of the inverse scattering transform. For vanishing boundary conditions, Zakharov and Shabat (1972) found that for long times the solution consists of a combination of envelope solitons and radiation modes, in analogy with the solution of the Korteweg-de Vries equation. For periodic boundary conditions, the solution is more complex. Linear stability analysis of a uniform wave train shows that close side bands grow exponentially in time in good qualitative agreement with the experimental results of Benjamin and Feir (1967) and Lake et al. (1977). For long times there is a considerable energy transfer from the carrier wave to the side bands.

Numerical modeling performed with a higher-order nonlinear Schrödinger equation can provide more information, as shown in the comparison with real data from field experiment collected in the WACSIS JIP (WAve Crest Sensor Intercomparison Study) done in the work of Liu et al. (2005), as well as in other studies that dealt with full-scale data (Slunyaev et al., 2005, 2013).

Although higher-order nonlinear equations such as the Dysthe equation are more accurate than the NLS (nonlinear Schrödinger) model (Shemer et al., 2002) in describing the evolution of groups of strongly nonlinear waves produced in the wave tanks, the studies of the properties of the nonlinear Schrödinger equation have been vital in understanding the conditions under which freak waves may occur (Onorato et al., 2001). One typical example explaining extreme waves is the work of Osborne (2000) where the solution of the onedimensional nonlinear Schrödinger equation with periodic boundary conditions is written as a "linear" superposition of stable modes, unstable modes, and their mutual nonlinear interactions. The stable modes form a Gaussian background wave field from which the unstable modes occasionally rise up and subsequently disappear again, repeating the process quasi-periodically in time.

In this study, the properties of the nonlinear Schrödinger equation will be analyzed to describe the evolution of various envelopes, and simulations of random waves in unidirectional sea states characterized by the JONSWAP power spectrum will be performed. Experiments carried out in the offshore basin of CEHIPAR (El Pardo Model Basin), Spain, are categorized into three groups according to the initial wave steepness parameter and are compared with numerical simulations as well. The influence of nonlinearity on the prediction of extreme wave heights is also investigated and some statistical characteristics of maximum wave height are presented on the basis of the initial Benjamin-Feir index (BFI). This complements similar experimental work and analysis performed in Onorato et al. (2006) and Mori et al. (2007), by using new experimental data.

This paper is organized as follows: in Sect. 2 a short review of basic theory and analytical formulae applied in this paper are given. Section 3 briefly introduces the facilities in the wave basin and the experimental data, and Sect. 4 is devoted to the numerical simulation of spatial evolution of wave envelops of Gaussian and bichromatic waves. The exceedance distributions of wave height, from laboratory experiments and numerical simulations, in three typical sea states are compared in the first part of Sect. 5, where the theoretical models including linear and nonlinear are also presented. The second part of Sect. 5 reveals some statistics on the maximum wave heights and some useful conclusions are summarized in the last section.

\section{Theory}

The simplest weakly nonlinear model that describes the evolution of free waves is the so-called cubic Schrödinger equation, which has been derived from the Zakharov equation under the narrowband approximation (Zakharov, 1968) and represents a perfect framework in which the basic features of the modulational instability are contained. Working as a balance between dispersion and nonlinearity, the dimensional NLS equation in arbitrary depth, in a frame of reference moving with the group velocity, has the following form:

$$
\frac{\partial A}{\partial t}+i \alpha \frac{\omega_{0}}{8 k_{0}^{2}} \frac{\partial^{2} A}{\partial x^{2}}+i \beta \frac{\omega_{0} k_{0}^{2}}{2}|A|^{2} A=0,
$$

where $A$ is the complex wave envelope, $\omega_{0}$ and $k_{0}$ are the carrier wave frequency and corresponding wave number. $\alpha$ and $\beta$ are two coefficients that in general depend on the dimensionless water depth $k_{0} h$, and both tend to 1 as $k_{0} h \rightarrow \infty$ approaches infinity. The analytical forms of $\alpha$ and $\beta$ can be found in the book of Mei (1989).

In order to derive the BFI in a simple and instructive way, Eq. (1) is nondimensionalized in the following ways: 


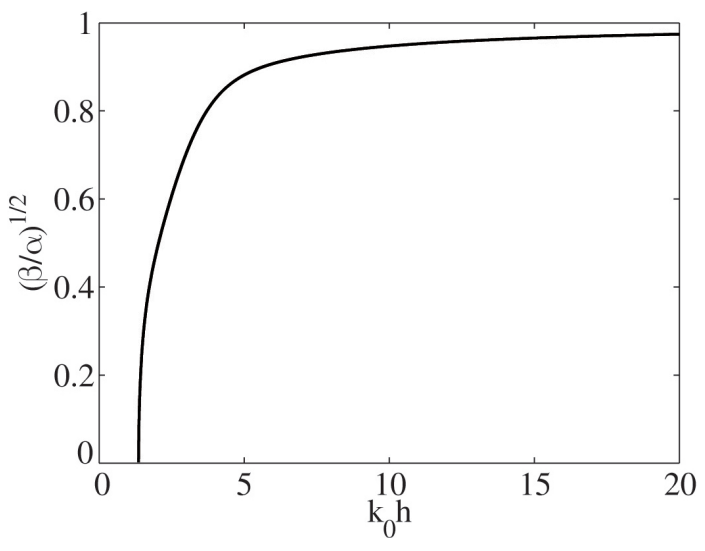

Fig. 1. Effect of finite water depth on the BFI.

$A^{\prime}=A / a_{0}, x^{\prime}=x \Delta K$ and $t^{\prime}=t\left(\Delta K / k_{0}\right)^{2} \alpha \omega_{0} / 8$, where $\Delta K$ and $a_{0}$ represent typical spectral bandwidth and wave amplitude, respectively, and thus reduces to

$\frac{\partial A}{\partial t}+i \frac{\partial^{2} A}{\partial x^{2}}+i\left(\frac{2 \epsilon}{\Delta K / k_{0}}\right)^{2} \frac{\beta}{\alpha}|A|^{2} A=0$,

where primes have been omitted for brevity. $\epsilon=\mathrm{a}_{0} \mathrm{k}_{0}$ is a measure of the wave steepness. The Benjamin-Feir index is now defined as the square root of the coefficient of nonlinear term:

$\mathrm{BFI}=\frac{2 \epsilon}{\Delta K / k_{0}} \sqrt{\frac{|\beta|}{\alpha}}$.

The term $\sqrt{\top} \beta \mid / \alpha$ is close to 1 as $k_{0} h$ tends to infinity and decreases as the water becomes increasingly shallow, and $\beta$ will become negative if the value of $k_{0} h$ is smaller than 1.36 as indicated in Fig. 1. In such case the modulational instability disappears and Stokes waves are stable to perturbations (Shemer et al., 1998; Onorato et al., 2001). Considering that time series are normally measured in the laboratory experiments, the term $\Delta K / k_{0}$ in the BFI is replaced by $2 \Delta \omega / \omega_{0}$ for infinite water depth. Due to a historical reason, a factor $\sqrt{2}$ is also added into Eq. (3), hence a random wave train tends to become unstable if BFI > 1 (Alber and Saffman, 1978).

For the purpose of better understanding the capability of the NLS equation in describing the evolution of propagating wave packets with a narrow spectrum, two simple but typical shapes of initial surface elevation are analyzed in the following numerical simulation. The first simulated series has a Gaussian shape of envelope and its initial surface elevation is

$\eta(t)=a_{0} \exp \left[-\left(\frac{t}{m T_{0}}\right)^{2}\right] \cos \left(\omega_{0} t\right)-16 T_{0}<t<16 T_{0}$,

where the carrier wave period $T_{0}=2 \pi / \omega_{0}$. The energy spectrum of Eq. (4) presents a Gaussian shape as well and its relative width at half maximum is given by
$\Delta \omega / \omega_{0}=\sqrt{2 \ln 2} /(2 m \pi)$. The value of the parameter $m$ is set to be 4.0 in this paper, so that all cases considered meet the condition $\Delta \omega / \omega_{0}=0.047<\epsilon$, thus satisfying the narrow spectrum assumption of the NLS equation.

In the second series of numerical simulations the bichromatic wave has been studied with the initial surface elevation in the following form:

$\eta(t)=a_{0} \cos \left(\omega_{0} / 20 t\right) \cos \left(\omega_{0} t\right)-15 T_{0}<t<15 T_{0}$.

The carrier wave frequency and the maximum amplitude in the simulation are identical to those with the shape given by Eq.(4). The spectrum of this kind of surface elevation is bimodal, with two equal-height peaks at $\omega=\omega_{0} \pm \Delta \omega$, where $\Delta \omega=\omega_{0} / 20$, satisfying the requirement of narrow spectrum approximation again (Shemer et al., 2002).

For the more realistic ocean environment, the initial condition for the numerical simulations is typical of sea states described by the JONSWAP (Joint North Sea Wave Project) power spectrum.

$S(\omega)=\alpha_{1} g^{2} \omega^{-5} \exp \left[-\frac{5}{4}\left(\frac{\omega_{0}}{\omega}\right)^{4}\right] \gamma^{\exp \left[-\left(\omega-\omega_{0}\right)^{2} /\left(2 \sigma_{0}^{2} \omega_{0}^{2}\right)\right],}$

where $\sigma_{0}=0.07$ if $\omega \leq \omega_{0}$ and $\sigma_{0}=0.09$ if $\omega>\omega_{0}$. Here $\omega_{0}$ is the peak frequency, $\gamma$ is the peak enhancement parameter, and $\alpha_{1}$ is the Phillips' constant related with the significant wave height $H_{\mathrm{s}}$. As $\gamma$ increases, the spectrum becomes higher and narrower around the peak frequency. In the present simulations, $\gamma=3$ and $\Delta \omega$ is estimated with halfwidth at half maximum of the computed wave spectrum and $\epsilon=k_{0} H_{\mathrm{s}} / 2$ (Onorato et al., 2006). The initial JONSWAP random surface elevation has been synthesized as sums of independent harmonic components, by means of the inverse fast Fourier transform of complex random Fourier amplitudes, which are prepared according to the "random realization approach" by using random spectral amplitudes as well as random phases (Onorato and Proment, 2011).

Some previous investigations (Hagen, 2002; Onorato et al., 2006; Cherneva et al., 2009; Toffoli et al., 2008a, b, $2010 \mathrm{a}, \mathrm{b}, 2011)$ suggest that the noticeably increasing frequency of occurrence of unusually large waves is accompanied by an increment on the coefficient of kurtosis $\lambda_{40}$, which has been related with the Benjamin-Feir index for narrowband long-crested waves at long times (Janssen, 2003).

$\lambda_{40}=\frac{\pi}{\sqrt{3}} B F I^{2}$,

where the BFI is stationary. Larger BFI means that nonlinearity dominates dispersion and apparently leads to a higher value of coefficient of kurtosis $\lambda_{40}$ in accordance with Eq. (7). Considering that BFI has a larger variability in its computation (Serio et al., 2005), it makes sense to use BFI as an initial parameter to indicate the chance of observing the freak waves and to take $\lambda_{40}$ as a critical parameter to describe 
Table 1. Locations of wave gauges.

\begin{tabular}{lrrrrrr}
\hline Gauge & 1 & 2 & 3 & 4 & 5 & 6 \\
\hline I Pos. (m) & 20 & 40 & 60 & 80 & 100 & 120 \\
II Pos. (m) & 30 & 50 & 70 & 90 & 110 & 130
\end{tabular}

the extreme wave height in the produced series. The statistics of the unusually large wave heights generated by modulation instability can be explained reasonably well by the theoretical approximations based on GC expansions (Bitner, 1980; Tayfun and Fedele, 2007; Mori and Janssen, 2006). Such approximation represents Hermite series expansions of distributions describing non-Gaussian random functions that are related to the stochastic structure of waves only through certain key statistics such as the coefficient of kurtosis of surface displacements. For narrowband long-crested waves, Mori and Janssen (2006) proposed a modified EdgeworthRayleigh (MER) distribution.

$E(h)=\exp \left(-\frac{h^{2}}{8}\right)\left[1+\frac{\lambda_{40}}{384} h^{2}\left(h^{2}-16\right)\right]$.

A more general form of third-order nonlinear model (GC) is given by Tayfun and Fedele (2007). Under a certain condition, i.e., $\Lambda \rightarrow \Lambda_{\text {app }} \equiv 8 \lambda_{40} / 3$ where $\Lambda=\lambda_{40}+2 \lambda_{22}+\lambda_{04}$, the GC model converges to the MER model. The cumulant coefficients are expressed in the same way as Tayfun and Lo (1990): $\lambda_{40}=\left\langle\eta_{1}^{4}\right\rangle / \sigma^{4}-3, \lambda_{22}=\left\langle\eta_{1}^{2} \hat{\eta}_{1}^{2}\right\rangle / \sigma^{4}-1, \lambda_{04}=$ $\left\langle\hat{\eta}_{1}^{4}\right\rangle / \sigma^{4}-3$ where $\sigma$ is the standard deviation of the free wave profile $\eta_{1}$ that is derived via inversion of the observational time series $\eta$ (Fedele et al., 2010). The bound waves could also be removed by band-pass filter (Onorato et al., 2005) or other procedures (Shemer et al., 2007). Evidently, $\eta_{1}$ is nonGaussian, but its crest and trough amplitudes have the same distribution. The symmetric amplification imposed on them is due to quasi-resonant interactions and reflected on $\Lambda$. If the third-order nonlinearity is negligible, i.e., $\lambda_{40} \cong 0$, Eq. (8) reduces to the Rayleigh exceedance distribution given by

$E(h)=\exp \left(-\frac{h^{2}}{8}\right)$.

\section{Facilities and experimental data}

The wave basin in CEHIPAR, Spain, is $152 \mathrm{~m}$ long, $30 \mathrm{~m}$ wide and $5 \mathrm{~m}$ deep as sketched in Fig. 2. The wave maker is located at one of the $30 \mathrm{~m}$ sides. The waves are produced by 60 flaps with independent motion. On the opposite side to the wave maker there is a wave beach that serves to absorb the incident wave energy. The wave maker can produce long- and short-crested sea states with up to $0.4 \mathrm{~m}$ significant wave heights and spectra of standard or arbitrary shape. The length scale of the laboratory experiments examined here is
Table 2. Parameters in different sea states.

\begin{tabular}{rrrrr}
\hline Case & $H_{\mathrm{S}}(\mathrm{m})$ & $T_{\mathrm{p}}(\mathrm{s})$ & $\varepsilon$ & Symbol \\
\hline 1 & 5 & 14 & 0.051 & \\
2 & 6 & 14 & 0.062 & \\
3 & 6 & 13 & 0.071 & \\
4 & 8 & 14 & 0.082 & Square \\
5 & 12 & 16 & 0.094 & \\
6 & 5 & 10 & 0.101 & \\
7 & 12 & 15 & 0.107 & \\
8 & 3 & 7 & 0.123 & \\
9 & 9 & 12 & 0.126 & \\
10 & 8 & 11 & 0.133 & \\
11 & 7 & 10 & 0.141 & Circle \\
12 & 3.5 & 7 & 0.144 & \\
13 & 6 & 9 & 0.149 & \\
14 & 11 & 12 & 0.154 & \\
15 & 8 & 10 & 0.161 & \\
\hline 16 & 4 & 7 & 0.164 & \\
17 & 12 & 12 & 0.168 & \\
18 & 7 & 9 & 0.174 & \\
19 & 9 & 10 & 0.181 & Triangle \\
20 & 11 & 11 & 0.183 & \\
21 & 6 & 8 & 0.188 & \\
22 & 12 & 11 & 0.199 & \\
23 & 5 & 7 & 0.205 & \\
\hline & & & & \\
\hline & & &
\end{tabular}

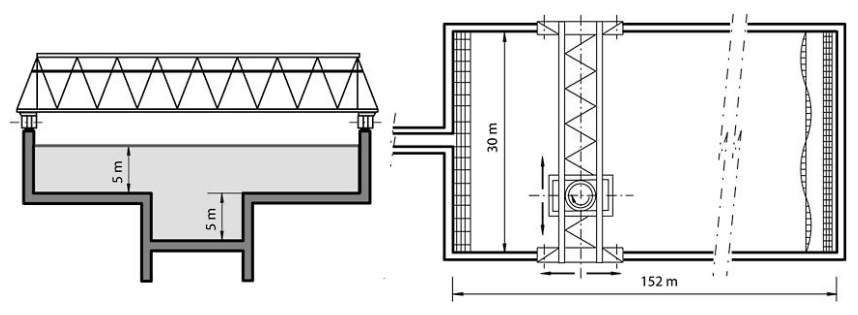

Fig. 2. Layout of the CEHIPAR wave basin.

$1: 40$. The waves generated for this study are long-crested and are registered by six capacitance wave gauges situated in the midline of the basin. Each experiment with the same initial conditions is carried out two times. For the second experiment, the gauges are moved $10 \mathrm{~m}$ downstream and repeat the same realization. The detailed gauge locations are listed in Table 1.

In this study the spectrum generated at the wave maker is unidirectional. Each experiment uses different sets of random phases but the variance of amplitudes is such that the spectrum of waves generated at the wave maker represents at full scale a JONSWAP spectrum characterized with peakenhancement factor $\gamma=3$ and Philips parameter $\alpha_{1}$ in dependence of the produced significant wave heights. 


\section{Wave envelopes}

In this section, the transformation of deterministic wave groups is investigated in deep water by the numerical solution of the simplest nonlinear model, i.e., the NLS equation which can reproduce the Benjamin-Feir instability. The initial wave envelopes are derived with the Hilbert transform of the surface elevations expressed in Eqs. (4) and (5), and depicted in Fig. 3, respectively. The generated surface elevations are normalized by their corresponding maximal wave amplitudes in the initial conditions. For economy of space, only three results from cases 4,13 and 23 in Table 2 are presented from top to bottom in Fig. 3, where the spatial evolution tendencies of envelopes are typical since they are from different sea state groups.

In the low sea state, e.g., Fig. 3a and d, no significant variation of the wave envelope along the tank is observed either in the Gaussian wave or in the bichromatic wave for the reason that the modulation instability is weak as the initial steepness is small.

In the moderate sea state, e.g., Fig. $3 b$ and e, they show the similar evolution speed but different tendency. For the envelope pulse, the initial Gaussian wave group adjusts its shape and width to become a fundamental soliton with oscillatory tails and attains a "sech" profile. The related theoretical predictions (Zakharov and Shabat, 1972) were tested experimentally by Yuen and Lake (1975). For the case of the evolution of a nonlinear continuous wave train, the amplitude does not grow exponentially for all time, but instead grows to a maximum value and then decreases in amplitude for later times and most of them repeat this oscillation periodically over time (Fermi-Pasta-Ulam recurrence). This modulation and demodulation process has been verified in the experiments (Lake et al., 1977) and the oscillatory unstable modes of this type are often referred to as breathers. In our simulation, this is demonstrated by a periodically repeated pulse train for the specified bichromatic wave envelope.

In the severe sea state, e.g., Fig. $3 \mathrm{c}$ and f, the same variation could be observed but with a higher evolution speed and a steeper envelope due to the stronger modulation instability. It needs to be stressed that for long distances the Gaussian envelope displays a periodic variation more or less like the continued series in the presence of stronger nonlinearity. Moreover, the symmetry of the initial condition is conserved in all cases, which obviously contradicts with the reality, but the envelope modulation is quantitatively correct, which has been confirmed many years ago. Thus the NLS equation could be applied in the analysis of extreme wave height distribution considering that it is capable of describing the evolution of the wave packet.
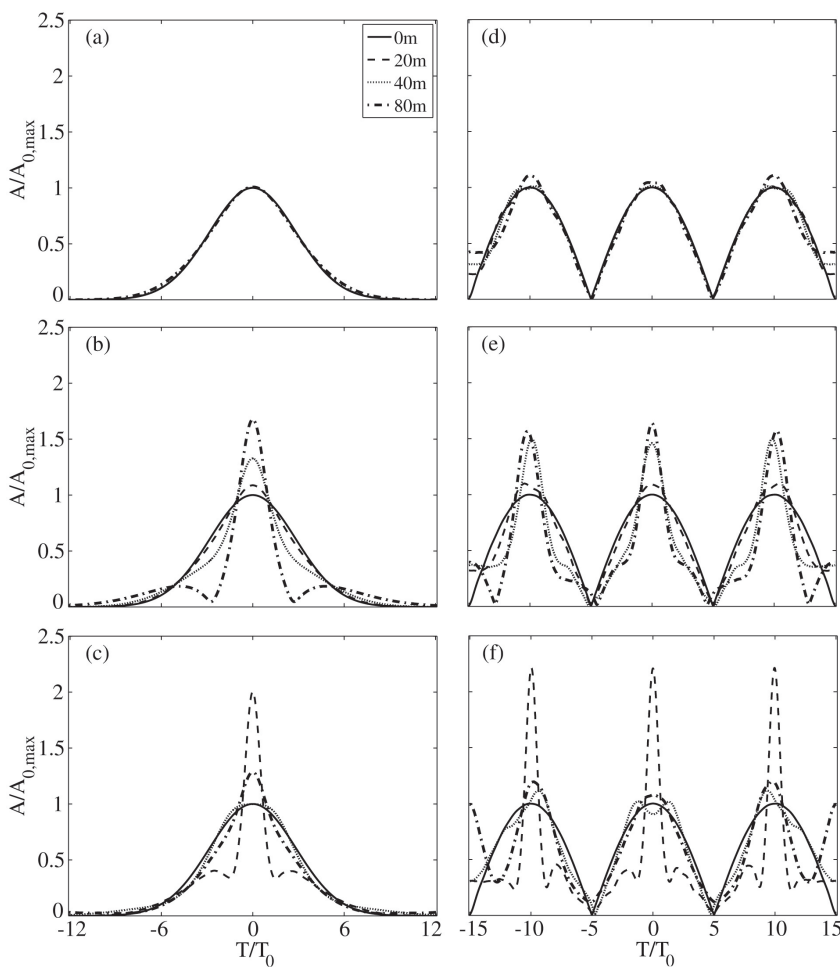

Fig. 3. Spatial variations of wave envelopes in three typical sea states. (a-c) are from Gaussian waves and (d-f) are from bichromatic waves. The three rows of panels correspond to the smooth, moderate and severe sea states, respectively.

\section{Comparisons}

\subsection{Exceedance distribution}

In general, $\Lambda$ is smaller than $\Lambda_{\text {app }}$ and, as pointed out by Cherneva et al. (2013), the difference will be slightly enlarged as the wave steepness increases. However, in Fig. 4 $\Lambda$ is almost equal to $\Lambda_{\text {app }}$ in all sea states not only in the simulation but also in the experiment where the bound waves have been removed. Thus it could be concluded that the small discrepancy between $\Lambda$ and $\Lambda_{\text {app }}$ is mainly due to the Stokes contribution and supports the fact that the strong deviation of coefficient of kurtosis from Gaussian behavior is in principle the result of modulation instability, i.e., a quasi-resonant four-wave interaction process that takes place near the peak of the spectrum (Onorato et al., 2005). Furthermore, it is obvious that larger $\Lambda$ appears in the case with larger initial BFI and the numerical simulations overestimate the experiments, especially in the third group of severe sea states.

With reference to the above discussion and that $\Lambda \approx \Lambda_{\text {app }}$, the MER model will make no difference to the GC model in the prediction of extreme wave heights; the MER model is adopted for the study in this paper. To have sufficiently good statistics, the exceedance probability of wave heights presented in Fig. 5 is based on both zero up-crossing and 


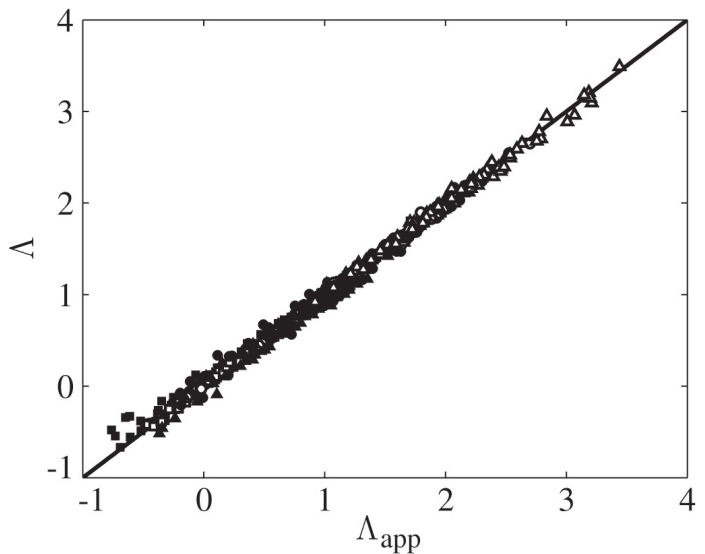

Fig. 4. Relationship between $\Lambda$ and $\Lambda_{\text {app }}$.

down-crossing waves. Due to the limitation in space, the comparison will only focus on three locations that could represent the initial, intermediate and end stages of wave evolution and correspond to the three columns in Fig. 5, respectively. From top to bottom, the sea states become more and more severe and come from three typical cases listed in Table 2. Meanwhile, for clarity in graphics, the empirical exceedance distributions are compared with the theoretical predictions of the linear Rayleigh distribution in Eq. (9) and the third-order MER model in Eq. (8) as well.

Under the low sea state, e.g., Fig. 5a-c, the numerical simulation and experimental data are in good agreement. Since the initial wave steepness is so small in the first group that the nonlinearity is negligible and the wave surface elevation approximates to be Gaussian distributed. As a result, the thirdorder nonlinear MER model reduces to Rayleigh statistics along the wave tank as anticipated.

As for the moderate sea state, e.g., Fig. 5d-f, the NLS equation also simulates the experiment reasonably well except for the initial stage where the wave height in the experiment is still Rayleigh distributed but the numerical result has achieved a fully developed condition earlier due to no energy dissipation in the simulation. As the wave propagates downstream in the basin, modulation instability will significantly work on the evolution process, as reflected by the perfect fit of the third-order MER model to the larger wave height distribution. What needs to be reminded is that the number of waves in the time series also plays a significant role in the prediction of extreme wave heights, particularly in longterm evaluations (Mori and Janssen, 2006; Cherneva et al., 2011; Zhang et al., 2013). Further comparison of MER model with a large amount of data from a higher-order nonlinear Schrödinger equation can be found in the work of Gramstad and Trulsen (2007).

In the most severe sea state, e.g., Fig. 5g-i, the same conclusion can be drawn that the NLS equation still captures the main characteristics of extreme wave heights, particularly in
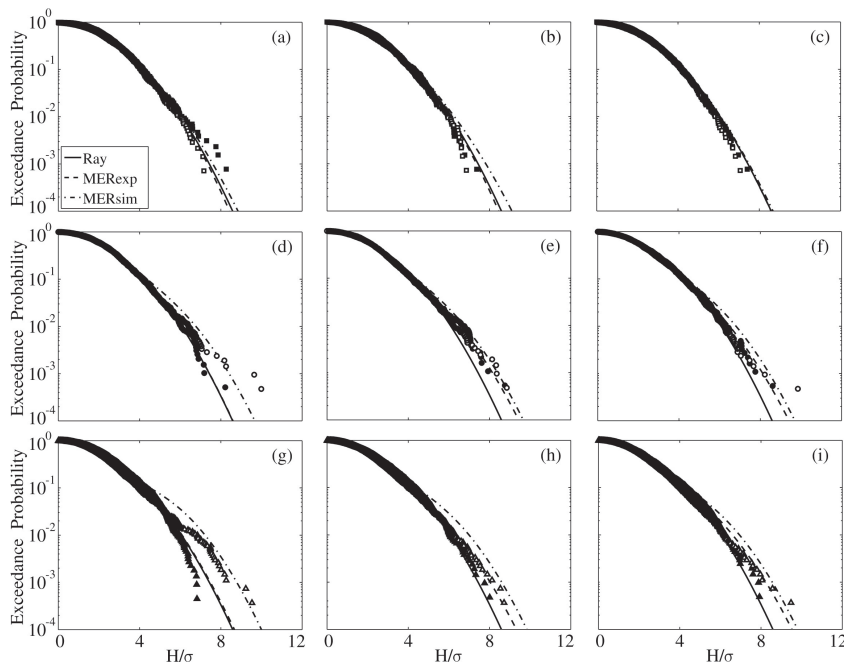

Fig. 5. Exceedance distributions of scaled wave heights. The three rows correspond to three typical sea states listed in Table 2, i.e., case 4 , case 13 and case 23 . The three columns present the results obtained from Gauges set at 20,80 and $120 \mathrm{~m}$ away from the wave maker, respectively. The solid line, dash line and dot-dash line mean Rayleigh distribution, MER models in experiment and in simulation in sequence. The full and empty marks still have the same meaning as before.

the intermediate and end stages of the evolution process, and the pronounced deviation at the beginning stage can be explained in the same manner as before. It is also detected that the numerical simulation presents larger wave heights than the experiments, which definitely attribute to the energy dissipation such as wave breaking in reality (Bitner-Gregersen and Toffoli, 2012). In other words, to give an exact description of the tail of the exceedance probability, the influence of wave breaking must be considered.

\subsection{Statistics on maximum wave heights}

The relationship between the coefficient of kurtosis and BFI is depicted in Fig. 6. As expected, the theoretical function (solid line) represented by Eq. (7) overestimates the results both in experiments and in simulations for the reason that the analytical expression is derived for the nonlinear steady state at infinity while the data points are from all wave gauges, for most of which the nonlinearity is not fully developed. Moreover, in laboratory experiments, the coefficient of kurtosis is sensitive to wave breaking in high sea states, which definitely leads to a further deviation from the theoretical prediction as we can see (the full symbols) in Fig. 6. These conclusions are in conformity with those derived in MARINTEK (Norwegian Marine Technology Research Institute; Zhang et al., 2013).

Based on a large number of abnormal waves registered in the Sea of Japan, Tomita and Kawamura (2000) obtained an appreciable correlation between the scaled maximum wave 


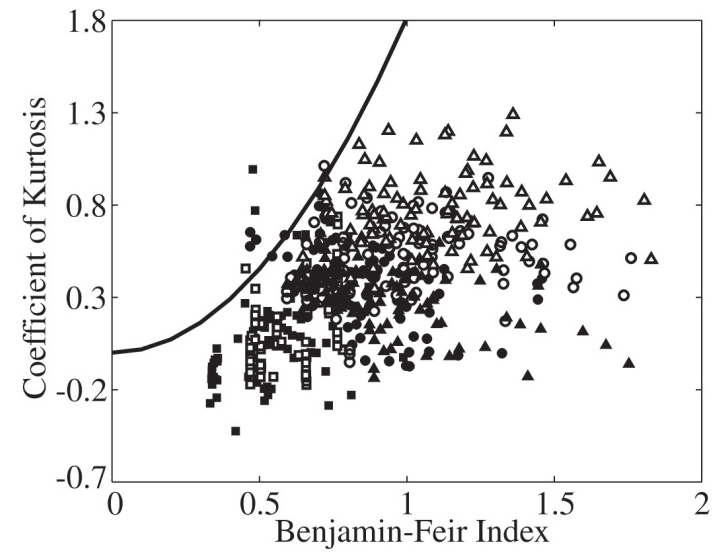

Fig. 6. Coefficient of kurtosis vs. BFI.

height and the coefficient of kurtosis. After analyzing all sea state records during the storm of November 1997 in North Alwyn and Draupner's storm in the beginning of 1995, a linear regression model was derived by Guedes Soares et al. (2003). As shown in Fig. 7, the same conclusion can be seen clearly in the present experiments and simulations. Now it is confirmed again that the behavior of some statistical quantities such as $\lambda_{40}$ could work as an indicator on the presence of extreme events in the time series (Guedes Soares et al., 2004). In this sense, the coefficient of kurtosis is thought to be the representative statistical measure with respect to the probability of occurrence of abnormal waves (Hagen, 2002). Meanwhile, it is observed again that the simulated results are larger than those in the experiments, particularly in the case with larger initial BFI. As we have explained before, this discrepancy is due to the nonbreaking phenomenon which leads to no energy dissipation and thus permits the formation of extreme large waves.

As a result from the interest in understanding the rogue wave generation, the maximum achievable wave height during the evolution of an unstable wave train has been investigated in the past. The first experimental study was made by $\mathrm{Su}$ and Green (1984), who tried to describe the steepness of the maximum wave as a function of the initial steepness of the wave train. Moreover, their results were further compared with the cubic NLS solution by Tanaka (1990) who had shown that the simulation generated much higher maximum wave steepness than the tank experiment, and were also compared with another tank experiment carried out in Tokyo by Waseda (2005), who showed a systematic deviation from Su's result and gave a higher value due to the controlled perturbations. In our experiments, the maximum wave heights are computed from zero up-crossing and down-crossing waves, respectively. Thus four-scaled maximum wave heights could be derived from the two series for each sea state, and their mean value is displayed in Fig. 8. It is very interesting that the maximal scaled wave height appears in the moderate sea state where the initial steepness

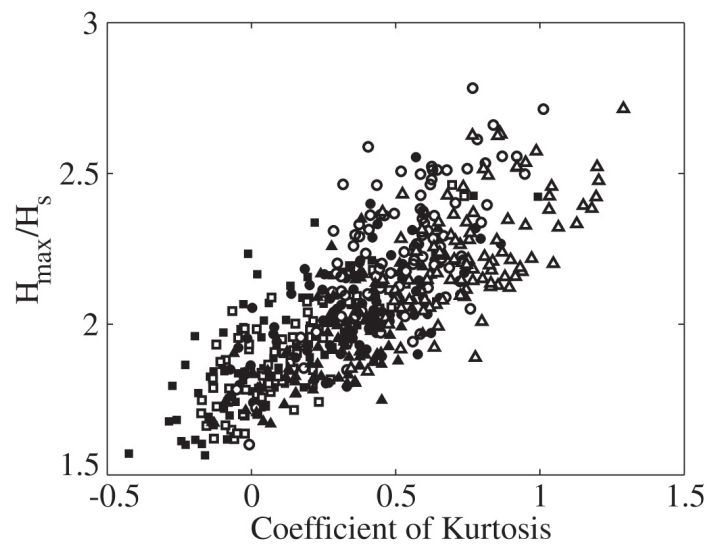

Fig. 7. Scaled maximum wave height vs. coefficient of kurtosis.

is $\varepsilon \approx 0.14$ rather than in the severe sea state. This variation is consistent with that observed in Su and Green's experiment (1984). The numerical simulation also presents the same tendency although a little overestimation is observed in moderate and severe sea states. Considering that there is no energy dissipation in the numerical simulation, this change should be attributed in part to the complicated nonlinear effect.

The same procedure of processing wave data is adopted in Fig. 9 where the relationship between steepness of maximum wave height and initial Benjamin-Feir index is manifested. In our experiment a similar variation to that of $\mathrm{Su}$ and Green's (1984) and Waseda's results (2005) occurs, despite the difference in the wave system (continuous spectrum vs. three-waves system). Our numerical results are also consistent with those derived from simulations of higherorder nonlinear equations, i.e., Dysthe and Zakharov equations (Waseda, 2005). Apparently, in Fig. 9, a good agreement between simulation and experiment could be detected except for the severe sea state and this discrepancy is mainly due to energy dissipation in the form of wave breaking. Moreover, many observations have proved that the maximum wave steepness (Michell, 1893) known as the Miche-Stokes limit, which is close to 0.1429 in deep water and depicted by the dashed line in Fig. 9, could be exceeded (Toffoli et al., 2010b), but the mean steepness of maximum wave height in our laboratory experiment does not exceed the Stokes limit. Thus it reveals that the change of wave shape is more related to the reduction of the wave period rather than to the increase of wave height (Toffoli et al., 2010b).

\section{Conclusions}

This paper, considers the capability of the NLS equation in describing the evolution of propagating wave packets that have a narrow spectrum. The influence of nonlinearity on the prediction of extreme wave heights in different random sea states is also investigated. The high-level agreement between 


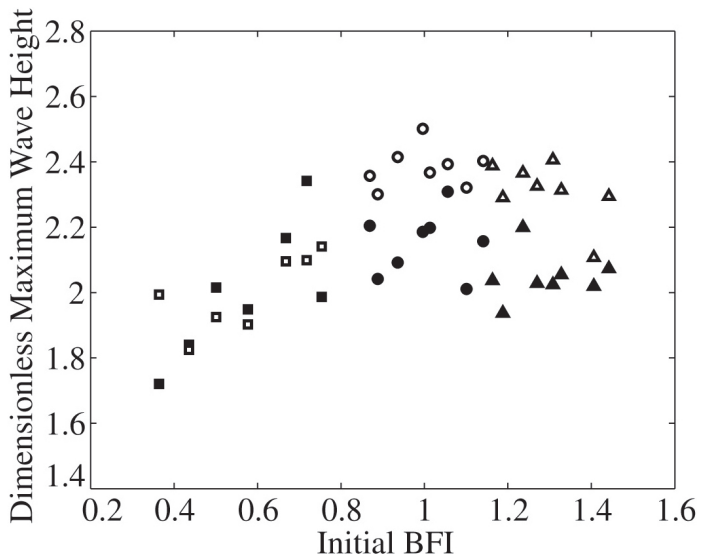

Fig. 8. Dimensionless maximum wave height vs. initial BFI.

numerical simulations, governed by the dynamics of the NLS equation, and the laboratory experiments carried out in the wave basin of CEHIPAR, provides another validation for the nonlinear Schrödinger equation in describing the formation of extreme waves.

However, the numerical simulations overestimate the experimental results particularly in the group of severe sea state for the reason that the NLS equation could not model wave breaking. This kind of limitation, which also the higherorder spectral method (HOSM) has, is observed in other studies as well (Bitner-Gregersen and Toffoli, 2012). Nevertheless, it still catches the main characteristics of the extreme waves and provides an important physical insight of their formation.

The speed and type of the evolution of the wave packet strongly depends on the initial conditions. The envelope pulse will eventually disintegrate into a definite number of envelope pulses or solitons that are stable to collisions (Zakharov and Shabat, 1972). In the absence of dissipative effects, the end state of the evolution of a nonlinear wave train in deep water is neither random nor steady, but is a series of periodically recurring states (FPU recurrence).

Without the influence of bound waves, third-order GC and MER models make no difference in prediction of extreme wave heights in that $\Lambda$ is almost equal to $\Lambda_{\text {app }}$ in all sea states not only for laboratory experiments but also for numerical simulations. To be more precise, the MER model tends to the linear Rayleigh distribution due to the insignificant nonlinearity in the low sea state; as the evolution of waves approaches the fully developed condition in the moderate sea state, the third-order MER model can describe the larger wave heights reasonably well; in the most severe sea state MER model still works but is strongly affected by the serious wave breaking in the experiment.

As expected, the relationship between the coefficient of kurtosis and BFI is overestimated by Eq. (7) considering that the analytical expression is derived for the nonlinear steady state at infinity while the data points are from all wave

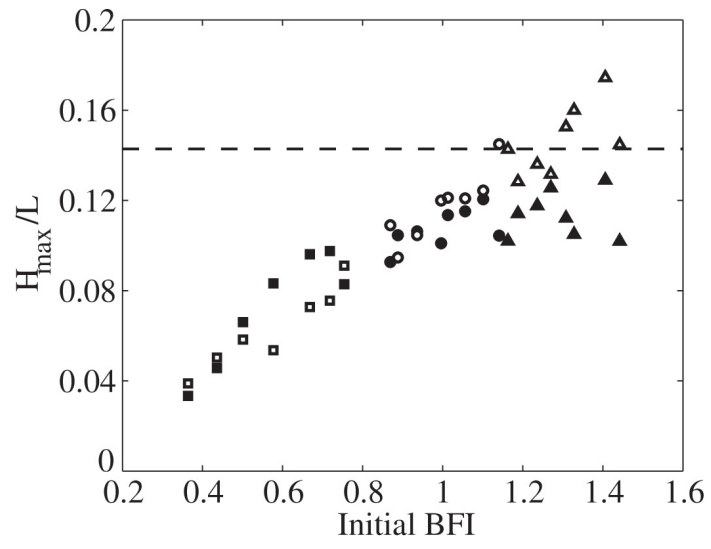

Fig. 9. Steepness of maximum wave height vs. initial BFI.

gauges. The scaled maximum wave height presents a highly correlated relationship with the coefficient of kurtosis. Thus to a certain degree, $\lambda_{40}$ can give an indication of the presence of extreme events in the time series.

It is noted that the maximal-scaled wave height appears in the moderate sea state where the initial steepness is $\varepsilon \approx 0.14$ rather than in the severe sea state (Su and Green, 1984), and the numerical simulation also presents the same tendency. Considering that there is no energy dissipation in the numerical simulation, this phenomenon should be attributed in part to the complicated nonlinear effect. For the severe sea state represented by larger initial BFI, the steepness of maximum wave height is normally large. Since the mean steepness of maximum wave height in our laboratory experiment does not exceed the Stokes limit, while the exceedance has been reported in many research works, it indicates that the change of wave shape is more related to the reduction of the wave period rather than to the increase of wave height (Toffoli et al., 2010b).

It should be also mentioned that real ocean waves are not long-crested and directional spread may play an important role in determining the statistical properties of the surface elevation as described in Onorato et al. (2009), Mori et al. (2011) and Toffoli et al. (2010a).Numerical modeling performed with a higher-order nonlinear Schrödinger equation can provide more information, as the comparison with real data from field experiments collected in the WACSIS JIP by Liu et al. (2005).

Acknowledgements. The present work has been performed within the project EXTREME SEAS (www.mar.ist.utl.pt/extremeseas/), Design for Ship Safety in Extreme Seas, which has been partially financed by the European Union through its 7th Framework Programme under contract SCP8-GA-2009-24175. The experiments in CEHIPAR that have been used in this study have been conducted by Adolfo Maron. M. Onorato thanks B. Giulinico for interesting discussions.

Edited by: E. Bitner-Gregersen

Reviewed by: three anonymous referees 


\section{References}

Alber, I., and Saffman, P.: Stability of random nonlinear deep-water waves with finite bandwidth spectra, Tech. Rep. 31326-6035RU-00, TRW Defense and Space System Group, 1978.

Benjamin, T. B., and Feir, J. E.: The disintegration of wave trains on deep water. Part 1. Theory, J. Fluid Mech., 27, 417-430, 1967.

Bitner (Bitner-Gregersen after marriage), E. M.: Nonlinear effects of the statistical model of shallow-water wind waves, Appl. Ocean Res., 2, 63-73, 1980.

Bitner-Gregersen, E. M., and Toffoli, A.: On the probability of occurrence of rogue waves, Nat. Hazards Earth Syst. Sci., 12, 751762,2012 , http://www.nat-hazards-earth-syst-sci.net/12/751/2012/.

Caponi, E. A., Saffman, P. G., and Yuen, H. C.: Instability and confined chaos in a nonlinear dispersive wave system, Phys. Fluids, 25, 2159-2166, 1982.

Cherneva, Z., Tayfun, M. A., and Guedes Soares, C.: Statistics of nonlinear waves generated in an offshore wave basin, J. Geophys. Res., 114, C08005, doi:10.1029/2009JC005332, 2009.

Cherneva, Z., Guedes Soares, C., and Petrova, P.: Distribution of wave height maxima in storm sea states, J. Offshore Mech. Arct. Eng., 133, 041601-1, 2011.

Cherneva, Z., Tayfun, M. A., and Guedes Soares, C.: Statistics of waves with different steepness simulated in a wave basin, Ocean Eng., 60, 186-192, 2013.

Davey, A.: The propagation of a weak nonlinear wave, J. Fluid Mech., 53, 769-781, 1972.

Fedele, F., Cherneva, Z., Tayfun, M. A., and Guedes Soares, C.: Nonlinear Schrödinger invariants and wave statistics, Phys. Fluids, 22, 036601, doi:10.1063/1.3325585, 2010.

Fonseca, N., Pascoal, R., Guedes Soares, C., Clauss, G. F., and Schmittner, C. E.: Numerical and experimental analysis of extreme wave induced vertical bending moments on a FPSO, Appl. Ocean Res., 32, 374-390, 2010.

Gramstad, O. and Trulsen, K.: Influence of crest and group length on the occurrence of freak waves, J. Fluid Mech., 582, 463-472, 2007.

Guedes Soares, C.: Probabilistic models of waves in the coastal zone, Advances in Coastal Modeling, edited by: Lakhan, C., Elsevier Science B, 6, 159-187, 2003.

Guedes Soares, C., Cherneva, Z., and Antão, E.: Characteristics of abnormal waves in North Sea storm sea states, Appl. Ocean Res., 25, 337-344, 2003.

Guedes Soares, C., Cherneva, Z., and Antão, E.: Abnormal waves during the hurricane Camille, J. Geophys. Res., 109, C08008, doi:10.1029/2003JC002244, 2004a.

Guedes Soares, C., Cherneva, Z., and Antao, E. Steepness, and Asymmetry of the Largest Waves in Storm Sea States, Ocean Engineering., 31, 1147-1167, 2004b.

Guedes Soares, C., Fonseca, N., and Pascoal, R.: Abnormal Wave Induced Load Effects in Ship Structures, J. Ship Res., 52, 30-44, 2008.

Hagen, Ø.: Statistics for the Draupner January 1995 freak wave event, In: Proceedings of the 21st International Conference on Offshore Mechanics and Arctic Engineering (OMAE'03), ASME Paper OMAE2002-28608, 2002.

Hasimoto, H. and Ono, H.: Nonlinear modulation of gravity waves, J. Phys. Soc. Jpn., 33, 805-811, 1972.
Hasselmann, K.: On the non-linear energy transfer in a gravity-wave spectrum, Part 1 - general theory, J. Fluid Mech., 12, 481-500, 1962.

Janssen, P. A. E. M.: Nonlinear four-wave interactions and freak waves, J. Phys. Oceanogr., 33, 863-884, 2003.

Lake, B. M., Yuen, H. C., Rungaldier, H., and Ferguson, W. E.: Nonlinear deep-water waves: theory and experiment, Part 2 - evolution of a continuous wave train, J. Fluid Mech., 83, 49-74, 1977.

Liu, J., Krogstad, H. E., Trulsen, K. Dysthe, K., and SocquetJuglard, H.: The statistical distribution of a nonlinear ocean surface, Int. J. Offshore and Polar Eng., 15, 168-174, 2005.

Longuet-Higgins, M.: On the statistical distribution of the heights of sea waves, J. Marine Res., 11, 245-266, 1952.

Longuet-Higgins, M.: The effect of non-linearities on statistical distribution in the theory of sea waves, J. Fluid Mech., 17, 459-480, 1963.

Mei, C. C.: The Applied Dynamics of Ocean Surface Waves, World Scientific, Singapore, 1989.

Michell, J. H.: On the highest waves in water, Philos. Mag., 5, 430437, 1893.

Mori, N. and Janssen, P.: On kurtosis and occurrence probability of freak waves, J. Phys. Oceanogr., 36, 1471-1483, 2006.

Mori, N., Onorato, M., and Janssen, P. A.: On the estimation of the kurtosis in directional sea states for freak wave forecasting, J. Phys. Oceanogr., 41, 1484-1497, 2011.

Onorato, M. and Proment, D.: Nonlinear interactions and extreme waves: Envelope equations and experimental results, in: Marine Technology and Engineering, edited by: Guedes Soares, C., Garbatov, Y., Sutulo, S., and Santos, T. A., Taylor and Francis Group, London, 135-146, 2011.

Onorato, M., Osborne, A., Serio, M., and Bertone, S.: Freak waves in random oceanic sea states, Phys. Rev. Lett., 86, 5831-5834, 2001.

Onorato, M., Osborne, A., Serio, M., and Cavaleri, L.: Modulational instability and non-Gaussian statistics in experimental random water-wave trains, Phys. Fluids, 17, 078101, doi:10.1063/1.1946769, 2005.

Onorato, M., Osborne, A., Serio, M., Cavaleri, L., Brandini, C., and Stansberg, C.: Extreme waves, modulational instability and second order theory: wave flume experiments on irregular waves. Europ. J. Mech. B/ Fluids, 25, 586-601, 2006.

Onorato, M., Cavaleri, L., Fouques, S., Gramstad, O., Janssen, P. A. E. M., Monbaliu, J., Osborne, A. R., Pakozdi, C., Serio, M., Stansberg, C. T., Toffoli, A., and Trulsen, K.: Statistical properties of mechanically generated surface gravity waves: a laboratory experiment in a three-dimensional wave basin, J. Fluid Mech., 627, 235-257, 2009.

Osborne, A.: Nonlinear instability analysis of deep water wave trains: computation of maximum heights of rogue waves. In: Proceedings of the 19th International Conference on Offshore Mechanics and Arctic Engineering (OMAE), ASME Paper OSU OFT-4033, 2000.

Petrova, P. and Guedes Soares, C.: Maximum wave crest and height statistics of irregular and abnormal waves in an offshore basin. Appl. Ocean Res., 30, 144-152, 2008.

Petrova, P., Cherneva, Z., and Guedes Soares, C.: On the adequacy of second-order models to predict abnormal waves, Ocean Eng., 34, 956-961, 2007. 
Serio, M., Onorato, M., Osborne, A., and Janssen, P. A. E. M.: On the computation of the Benjamin-Feir Index, IL NUOVO CIMENTO, doi:10.1393/ncc/i2005-10134-1, 2005.

Shemer, L. and Sergeeva, A.: An experimental study of spatial evolution of statistical parameters in a unidirectional narrow-banded random wave field, J. Geophys. Res., 114, C01015, doi:10.1029/ 2008JC005077, 2009.

Shemer, L., Kit, E., Jiao, H., and Eitan, O.: Experiments on Nonlinear Wave Groups in Intermediate Water Depth, J. Waterway, Port, Coastal, and Ocean Eng., 124, 320-327, 1998.

Shemer, L., Kit, E., and Jiao, H.: An experimental and numerical study of the spatial evolution of unidirectional nonlinear waterwave groups, Phys. Fluids, 14, 3380-3390, 2002.

Shemer, L., Goulitski, K., and Kit, E.: Evolution of wide-spectrum wave groups in a tank: An experiment and numerical study, Europ. J. Mech. B, 26, 193-219, 2007.

Shemer, L., Sergeeva, A., and Liberzon, D.: Effect of the initial spectrum on the spatial evolution of statistics of unidirectional nonlinear random waves, J. Geophys. Res., 115, C12039, doi:10.1029/ 2010JC006326, 2010.

Slunyaev, A., Pelinovsky, E., and Guedes Soares, C.: Modeling Freak Waves from the North Sea, Appl. Ocean Research., 27, 12-22, 2005.

Slunyaev, A., Pelinovsky, E., and Guedes Soares, C.: Reconstruction of Extreme Events Through Numerical Simulations, J. Offshore Mechan. Arctic Engin., 136, 011302, doi:10.1115/1.4025545, 2013.

$\mathrm{Su}, \mathrm{M}$. Y., and Greeen, A. W.: Coupled Two-dimensional and 3dimensional instabilities of surface gravity-waves, Phys. Fluids, 27, 2595-2597, 1984.

Tanaka, M.: Maximum amplitude of modulated wave train, Wave Motion, 12, 559-568, 1990.

Tayfun, M. A. and Fedele, F.: Wave-height distributions and nonlinear effects, Ocean Eng., 34, 1631-1649, 2007.

Tayfun, M. A. and Lo, J.: Nonlinear effects on wave envelope and phase, J. Waterway, Port, Coastal and Ocean Eng., 116, 79-100, 1990.

Toffoli, A. and Bitner-Gregersen, E. M. : Extreme and rouge waves in directional wave fields, Open Ocean Eng. J., 4, 24-33, 2011.
Toffoli, A., Bitner-Gregersen, E., Onorato, M., and Babanin, A. V.: Wave crest and trough distributions in a broad-banded directional wave field, Ocean Eng., 35, 1784-1792, 2008a.

Toffoli, A., Onorato, M., Bitner-Gregersen, E., Osborne, A. R., and Babanin, A. V.: Surface gravity waves from direct numerical simulations of the Euler equations: a comparison with second-order theory, Ocean Eng., 35, 367-379, 2008 b.

Toffoli, A., Gramstad, O., Trulsen, K., Monbaliu, J., BitnerGregersen, E. M., and Onorato, M. :Evolution of weakly nonlinear random directional waves: laboratory experiments and numerical simulations, J. Fluid Mech., 664, 313-336, 2010 a.

Toffoli, A., Babanin, A. V., Onorato, M., and Waseda, T.: Maximum steepness of oceanic waves: Field and laboratory experiments, Geophys. Res. Lett., 37, L05603, doi:10.1029/2009GL041771, 2010b.

Tomita, H. and Kawamura, T.: Statistical analysis and inference from the in-situ data of the Sea of Japan with reference to abnormal and/or freak waves, in: Proceedings of the 10th International Offshore and Polar Engineering Conference (ISOPE), Seattle, USA, 116-122, 2010b.

Waseda, T.: Experimental investigation and applications of the modulational wave train. In: Proceedings of the Workshop on Rogue Waves, 12-15 December 2005, ICMS, Edinburgh, 2005.

Yuen, H. C. and Ferguson, W. E.: Relationship between BenjaminFeir instability and recurrence in the nonlinear Schrödinger equation, Phys. Fluids, 21, 1275-1278, 1978.

Yuen, H. C. and Lake, B. M.: Nonlinear deep water waves: theory and experiment, Phys. Fluids, 18, 956-960, 1975.

Zakharov, V. E.: Stability of periodic waves of finite amplitude on a surface of deep fluid, J. Appl. Mech. Tech. Phys., 9, 190-194, 1968.

Zakharov, V. E. and Shabat, A. B.: Exact theory of two-dimensional self-focusing and one-dimensional self-modulating waves in nonlinear media, Sov. Phys. JETP, 34, 62-69, 1972.

Zhang, H. D., Cherneva, Z., Guedes Soares, C., and Onorato, M.: Comparison of distributions of wave heights from nonlinear Schrödinger equation simulations and laboratory experiments, Proceedings of the 32nd International Conference on Ocean, Offshore and Arctic Engineering, Nantes, France, OMAE 201311633, 2013. 